Lepr Rev (1989) 60, 278-282

\title{
Do we need trials of agents alleged to improve healing of plantar ulcers?
}

\author{
H SRINIVASAN \\ Central JALMA Institute for Leprosy, Taj Ganj, AGRA-282001, \\ India
}

Accepted for publication 10 July 1989

\begin{abstract}
Summary The assumptions underlying trials of agents claiming to heal plantar ulcers 'faster' and 'better' are shown to be fallacious and it is pointed out that in most cases these ulcers fail to heal for lack of attention and not for want of a specific topical agent. Clinical trials in this area are difficult and are not worth the trouble as they do not add to our knowledge about these ulcers or their management in the clinic or in the field.
\end{abstract}

\section{Introduction}

Physicians working in leprosy are approached now and again with a request to carry out a trial of an agent, a chemical, biological or a synthetic preparation, to assess its value (to certify its usefulness would be more correct) in healing plantar ulcers in leprosy patients. As a consequence, we see periodic publications reporting the usefulness of some agent or the other in obtaining or accelerating healing of plantar ulcers, some even claiming that the test agent prevented or reduced recurrence of those ulcers. A variety of 'healing agents' have been advocated in this manner, leaving leprosy workers, doctors and others, rather confused and making them feel that they should also be using the 'latest' such 'healing agent' for successfully tackling the ulcer problem in their area. In this context it was felt necessary to examine the rationale of the claims made on behalf of these "healing agents' before accepting them. Further, it was also necessary to examine whether these trials, conducted as they have been, contribute in any way to the corpus of our knowledge about plantar ulcers, or, help us to take better care of them.

\section{Claims and fallacies}

The claims made on behalf of these 'healing' agents are: (i) they 'get' plantar ulcers healed; (ii) they clean up the ulcer better and thus help in healing; (iii) they make ulcers heal faster; or (iv) that the healing obtained with the agent is sounder and so fewer of the 'treated' ulcers will recur. Let us examine the soundness of these claims.

(i) Claim regarding 'getting' ulcers healed: It would appear that plantar ulcers are normally incapable of healing and the assistance of the 'healing agent' is necessary to induce or restore the power of healing. The fact is, as we all know from our experience, plantar ulcers nearly always heal 
provided they are attended to and healing is not interfered with. As far as we know, there is no gross defect in the healing process in leprosy patients either because of leprosy or because of denervation and so there is no real reason why these ulcers should not heal. It is only occasionally that one finds it impossible to get the ulcer healed and in such cases the reasons can usually be found, such as deep infection, poor ulcer bed, or, onset of pseudoepitheliomatous or malignant change. To state that an agent 'gets' the ulcer healed is therefore tantamount to saying that the agent does not interfere with healing and nothing more.

(ii) Claim that the agent cleans up the ulcer better and helps healing: This claim is usually advanced on behalf of antibiotic/antiseptic preparations, others which 'draw the fluid off' the ulcer by osmosis or other such physical forces (e.g. Magsulph, micronized plastic granules) and proteolytic enzyme preparations. The point is that in most cases the ulcer is clean to start with and should not be allowed to become dirty but kept clean by simple barrier dressings. As for dirty ulcers, there are simple and cheap ways of effectively cleaning them-thorough and frequent washing, irrigation, or soaking - and it is only rarely that a special agent will be required for this purpose. For ulcers with lots of dead tissue, freshly prepared EUSOL (12.5 g of boric acid crystals and $12.5 \mathrm{~g}$ of bleaching powder per litre of water) or even just bleaching powder in water may be used for it is one of the few preparations active even in the presence of necrotic tissue, and it will be far cheaper to use than any other cleanser.

Asfor antibiotics, as a general rule it is not advisable to use a systemic antibiotic locally, to avoid the possibility of the patient developing hypersensitivity and the infecting organisms developing resistance. Further, dirty ulcers often carry 'street infection'- - mixed bag of organisms, many of which are not susceptible to commonly used and cheaper antibiotics. Indiscriminate and routine use of antibiotics in the treatment of ulcers is to be strongly condemned because by such use they are of ten rendered ineffective or even dangerous just when the patient may be urgently in need of them. Antibiotics must be kept in reserve for such occasions and should be used only when there are systemic signs of infection, like fever and acute regional adenitis, indicating a failure of localization of infection. As for local antibiotics and antiseptics, their use is not essential as the ulcers can generally be cleansed by irrigation and drainage. Their routine use makes people feel 'safe' and they become lax in the other necessary measures of keeping the ulcer clean. Unfortunately, faith in the magic of medicine is still very strong among the public including many doctors and medical auxillaries and the use of drugs as magic remedies tends to make them ignore other essential precautions.

(iii) Claim that healing agent obtains 'better and quicker' healing: This slogan is an advertising trick which has unfortunately crept into medicine. 'Better and quicker than what?' should be our automatic response to such a claim. But having become used to slogans like 'Detergent Blank washes whiter!' and 'Toothpaste Blank Blank cleans your teeth better!!', we tend to accept this claim equally uncritically.

The following points need to be noted in respect of this claim. (i) We already have a method of healing an ulcer by the shortest time, in two weeks or less, in skin grafting, if that is what is wanted. I am not aware of any 'healing agent' which equals this efficacy. In addition, skin grafting provides epithelial covering from the time the graft is applied, that is, from day zero, which no healing agent does. The technique is not very difficult and the procedure can be carried out in any primary health centre having facilities for clean surgery. (ii) In my experience, an uncomplicated, reasonably clean ulcer of average size takes about five weeks to heal under conditions of limited walking, when kept covered with saline dressings or Vaseline gauze only, without a plaster cast or bed rest (unpublished data). Marginal shortening of healing time is of no practical significance even when the difference is statistically significant. To be of practical significance, the healing time should be consistently and substantially reduced, say, by about $50 \%$ or more. To the best of my knowledge none of the "healing agents' has been shown to be that effective. (iii) Healing can become defective and healing time can get prolonged in conditions like gross nutritional deficiencies (e.g. vitamin, protein or trace metal deficiencies), diabetes, uraemia, and malignancy. But, these are not common and the two most 
common causes for delayed healing of plantar ulcers in leprosy patients are (a) continued injury to the ulcer by unlimited and unprotected walking, and (b) deep or persistent infection. If these are taken care of, the ulcer will heal normally. 'Early' healing of plantar ulcers thus requires removal or mitigation of factors that interfere with healing.

(iv) Claim that healing is sounder and recurrences are reduced: This claim is usually only implicitly made, as a bonus benefit from using the 'healing agent', by stating there were fewer instances of recurrent ulceration during the period of observation, or, just mentioning that the scar was 'more supple', hinting at a lesser possibility of recurrence. The facts are (a) that not all plantar ulcers recur, and (b) that any plantar ulcer can recur so long as the original factors that led to ulceration (anaesthesia, plantar intrinsic paralysis and unrestricted or unprotected walking) continue to be operative. Unlike in cancer, reliable estimates of recurrence rates for various kinds of plantar ulcers in leprosy patients are not available and so we are not in a position to predict the probability of recurrent ulceration for different periods of time after obtaining healing. Many persons show interest in the problem of plantar ulcers only for the duration of the trial and are, therefore, not in a position to make proper estimates of probable recurrence based on experience. In view of all this, statements on prevention of recurrence do not carry conviction.

We must remember that plantar ulcers recur because (i) original conditions that led to ulceration in the first instance continue to operate, (ii) the scar is unable to withstand normal stresses of walking, (iii) excessive stresses fall on the scar, and (iv) because of flare up of latent infection. It follows that prevention of recurrent plantar ulceration requires a variety of actions such as (i) using protective foot-wear and limiting walking to within safe limits, (ii) minimizing scar formation and improving its quality, (iii) reducing the stresses on the scar, and (iv) eradicating infection. The quality of scars is improved by limiting tissue loss to the minimum, healing the ulcer early, local physiotherapy (gentle massage etc.,) and, where needed, by surgical revision. Reducing the stress on the scar will require (i) modifications in foot-wear, (ii) limiting walking to within safe limits, and (iii) surgical measures to correct evident and not so evident anatomical abnormalities and deformities which throw excessive stress on the scar.

It should be clear from the above that getting the ulcer healed and preventing its recurrence are quite different propositions and that the latter is a far more complex task than the former. In such a situation, to depend on a 'healing agent' for preventing recurrence is like relying on better management of injuries from automobile accidents for preventing such accidents.

\section{The trials-a critique}

The trials themselves pose a number of problems which are briefly touched upon below.

(i) Trials are laborious and expensive: Properly planning and carrying out a trial of a "healing agent' requires much time and labour. It needs to be a well thought out and properly controlled double-blind study. Matching controls have to be carefully selected taking a number of parameters into account, such as age, sex, body-weight, pressure at ulcer site, distances walked by the subjects, past ulcer behaviour, local condition of the ulcer, integrity of blood flow and many other compounding factors. This is a laborious task as anybody experienced in these procedures will aver. Accurate records have to be maintained, patients may have to be hospitalized, additional investigations carried out and these further increase work all round.

(ii) Reliability of data: All this trouble may be worthwhile if the information collected is reasonably reliable and correct. Since plantar ulcers are a chronic problem with numerous remissions and exacerbations, information collected about them is often quite inaccurate. Investigators, when they become aware of these deficiencies, tend to become cursory in data collection adding to the unreliability of the data collected.

(iii) Eliminating bias: This is a major problem. Double-blind trials are necessary but are not usually possible as the person who attends to the ulcers (and the patients as well) will know whether 
the 'healing agent' is being used or not, and many investigators do not seem to realize that that is sufficient to introduce bias. The subtle ways in which such bias works is incredible but true. Perusal of the literature shows that anybody who has tested any remedy or agent seems to have always found the test remedy/agent superior to the control agent/remedy in some way or other, using tables, graphs, photographs and $p$ values to support their claim! It is very much like the situation regarding non-steroid anti-inflammatory drugs and gastric irritation. Each new drug is heralded with the fanfare 'this has proved to be far more kind to the stomach than all the previous ones', citing well-conducted trial reports to support the claim. If all these claims were really true, by now the latest such drug should be causing hardly any gastric irritation, which is of course not the case. Evidently some bias has crept in somewhere, somehow.

The fact seems to be that when one tests a 'healing agent', bias is inevitably introduced, whether one is aware of it or not and better results are obtained with the trial agent. This is best exemplified by the Canadian experiment ${ }^{1}$ in which exposure to a non-functioning gadget (placebo device) healed bed-sores better because it was believed by the attendants to emit an invisible healing ray! (I will be happy to supply a copy of this report to any one who is interested). It is evident that extraordinary care has to be taken in conducting these trials, with each step meticulously planned and executed to eliminate bias. Results of trials conducted without such care and attention to details (unfortunately many reported trials fall into this category) help only to misinform the reader.

(iv) Relevance of results: Assuming a careful study did show that an agent healed plantar ulcers a little earlier than the control remedy, it is difficult to see how this information becomes relevant. As pointed out earlier, if a 'healing agent' is advocated for plantar ulcers, the results become relevant only, (i) if the healing time is substantially shortened (by 50\% or more) and not otherwise, and (ii) if it is shown that the use of that agent would make ulcer care in institutions or in the field operationally easier and cheaper. To show merely that the 'healing agent' helped to heal plantar ulcers does not mean anything, for, as pointed out earlier, it is extremely rare to find that one cannot get a plantar ulcer healed. However, some leprosy patients do have recalcitrant extra-plantar ulcers (like stasis ulcers in the leg) and there may be a case for trying 'healing agents' on them.

\section{Concluding remarks}

From the time Ambroise Pare (16th Century) substituted egg white for boiling oil for treating war wounds, the quest for wound healing agents has been going on and will probably continue as long as people keep getting wounds and burns. The literature in leprosy is strewn with reports of agents that have helped healing plantar ulcers 'quicker and better'. They include acriflavin, amino acids, amniotic membrane, bacitracin, cod-liver oil, coffee powder, collagen sheet, dapsone, Debrisan, Furacin, gentian violet, hydnocarpus oil, hydrogen peroxide, hyperbaric oxygen, Livoderm, Madecassol, mercurochrome, Neomycin, Novolep, phenytoin, placental extract, polymyxin, sulphonamides, uracil, Vasodilators, vitamins, zincsalts and zinc tape. The list is not exhaustive and there are probably a lot more. The variety of 'healing agents' shown to be effective only demonstrates that plantar ulcers heal provided they are attended to, and not the effectiveness or superiority of any of these remedies.

Barring the few permanently settled in institutions, all leprosy patients live in their homes and a proportion of them have plantar ulcers. Leprosy clinics can at the most get the ulcer cleaned and dressed once or twice a month. It is evident that plantar ulcers will have to be attended to and cared for in the homes of patients, by the patients themselves or by some members of their families. Therefore the primary need is for leprosy patients with plantar ulcers to know how to manage their ulcers properly in their own homes. For this they require to know why they got their ulcers, how they can keep them clean, covered and protected so that they will heal and how they should conduct themselves to prevent recurrence. They also need to be helped to put this knowledge into practice, as well as when they have failed in their own efforts to get the ulcer healed. The offering of supposed 
'healing agents', whether they are the latest technological wonders or traditional remedies, only helps to perpetuate the myth of magic remedies encouraging the patients and the others to rely on the agent and not on their own health practices which are the basic requirements to get plantar ulcers healed and prevent their recurrence.

It is evident that trials of 'healing agents' for plantar ulcers may, at best, provide information of dubious value but not knowledge. Promoters of these agents may be interested in getting a certificate from an authority that 'agent $\mathrm{X}$ heals plantar ulcers better and faster', but that is no reason why people in authority should waste their efforts and oblige them.

\section{Reference}

${ }^{1}$ Fernie GR, Dornan J. The problems with clinical trials with new systems for preventing or healing decubiti, in Bed Sore Biomechanics, Kenedi RM, Cowden JM, (eds), London, Macmillan, 1976. 